\title{
Smooth and Inclined Surface Locomotion and Obstacle Scaling of a C-Legged Miniature Modular Robot
}

\author{
Nima Mahkam ${ }^{1}$, Talip Batuhan Yılmaz, and Onur Özcan ${ }^{2}$ \\ Bilkent University, Department of Mechanical Engineering, Ankara, Turkey
}

\begin{abstract}
This work investigates the locomotion of a modular C-legged miniature robot with soft or rigid backbones on smooth, rough, and inclined terrain. SMoLBot-C is a $\mathrm{C}-$ legged miniature robot with soft or rigid backbones and foldable modules. The robot's climbing capabilities with soft and rigid $\mathrm{C}$-legs and different backbones on rough terrain with obstacles and the robot's mobility on an inclined surface are compared. Our results show that the C-legged robot with soft legs and soft backbones can climb up to a higher obstacle, and walk on surfaces with higher inclination angles compared to the same robot with rigid legs and backbones, regardless of the number of modules (legs). Additionally, a velocity comparison study using SMoLBot-C operating at two different gaits is conducted. The results show that the robot with soft legs and compliant-I backbones operating with trot gait possesses the highest velocity compared to the other robots with similar leg numbers. Moreover, the effect of a compliant tail on the robot's locomotion on smooth and rough terrains is investigated, where the results show that the robot with the compliant tail is capable of walking on surfaces with higher inclination angles compared to the same robot without a tail. Furthermore, adding a tail to the two-legged SMoLBot-C doubles the maximum scalable obstacle height; the robot with a tail can climb up an obstacle 2 times higher than a module's height. Locomotion analysis in this manuscript provides a better insight into $\mathrm{C}$-legged miniature robots' locomotion with soft or rigid legs while the modular connections' structural stiffness varies from rigid to soft.
\end{abstract}

Index Terms-Soft Robot Materials and Design, Cellular and Modular Robots, Legged Robots.

\section{INTRODUCTION}

Miniature robots have many advantages, such as low manufacturing cost and rapid production. Additionally, they possess high maneuvering capabilities with agile locomotion that make these robots a nice candidate for search and rescue missions, performing explorations in complex areas, and inspection [1], where macro-scale robots fail to reach. Additionally, studies on the miniature robots' locomotion have shown that the maneuverability and the robustness of these small-scaled robots can be further improved by assembling different leg designs to miniature robots such as C-shaped legs [2], [3].

Various production techniques for miniature robots have been investigated by researchers such as MEMS fabrication [4], creating multilayer composite structures with smart composite microstructure (SCM) [5]-[8], 3D printing [9] and multi-material 3D printing (MM3P) [10]. SMoLBot$\mathrm{C}$, a modular miniature robot with soft or rigid backbones

All authors are with Department of Mechanical Engineering, Bilkent University, Ankara, Turkey. ${ }^{1}$ nima.mahkam@gmail.com, ${ }^{2}$ onurozcan@bilkent. edu.tr
(Fig. 1), uses the same origami-inspired fabrication method similar to [11]. The foldable module's fabrication starts with the laser cutting of an acetate sheet and then folding the two-dimensional design into a three-dimensional complex configuration [12], [13]. These foldable modules provide housings for 2 DC motors, a single battery, and two locking mechanisms. SMoLBot-C uses $\mathrm{C}-$ shaped legs that are 3D printed from Polylactic Acid (PLA) or molded from Polydimethylsiloxane (PDMS). C-shaped legs offer excellent opportunities to the miniature modular robots by improving maneuverability and mobility on rough terrains with obstacles or inclined surfaces [14], [15]. The locomotion of the miniature robots is significantly affected by small changes of the feet-ground contact forces. C-shaped legs form a larger contact with the surface than the legs with pointy feet and construct a better continuous feet-ground contact improving the miniature robot's mobility on a variety of rough and smooth terrains.

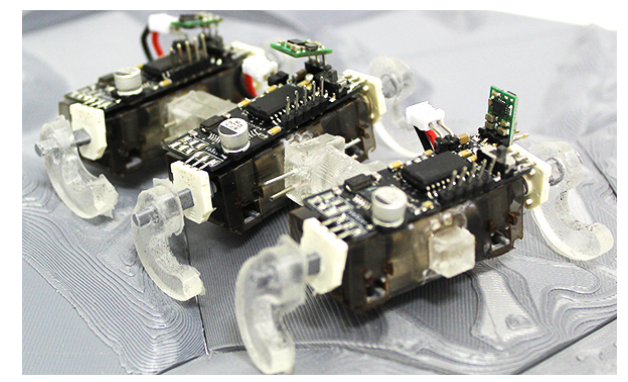

Fig. 1. C-Legged Soft Modular Robot (SMoLBot-C).

This work's contributions are the locomotion analyses investigating the effect of soft and rigid legs on the multilegged robot's obstacle climbing capabilities and inclined surface mobility while different backbones are assembled on the robot. As another contribution, a velocity comparison of SMoLBot-C with different modular connections (soft or rigid) and two types of $\mathrm{C}$-shaped legs is conducted to extend the locomotion analyses to smooth terrain. Furthermore, a compliant tail is added to the robot's rear module, and its effects on the robot's mobility are investigated. Results indicate that the robot with soft legs and soft backbones possesses the highest velocity among other robots, and the robot with a combination of compliant-T backbone, soft legs, and a compliant tail is capable of operating on a surface with $40^{\circ}$ inclination. In contrast, a rigid backbone rigid leg and no-tail robot can only climb a surface with a maximum $13^{\circ}$ inclination angle. 


\section{Design of SMoLBot-C}

\section{A. Body, Backbone and Controller Design}

SMoLBot-C is the C-legged version of SMoLBot [11], where the modules are cut and folded from flexible A4-size cellulose acetate sheets, and soft backbones and soft legs are molded from PDMS. Each robot module is $16.75 \mathrm{~mm}$ long, $44.5 \mathrm{~mm}$ wide, and $15 \mathrm{~mm}$ high, and weighs 12 grams. Three different backbones are used in SMoLBot-C experiments; rigid, compliant(T), and compliant(I) backbones (the backbone dimensions and stiffness values can be found in [11]). Rigid backbones are PDMS blocks with metal insertions possessing the highest bending and torsional stiffnesses, compliant( $\mathrm{T})$ backbones are rectangular beams that allow only torsional deflection, and compliant(I) backbones possess the minimum stiffnesses among the rest of the backbones that add 3 degrees of freedom (3-DOF) to the system. Each module has a PCB to control each leg independently. The detailed version of the module design, PCB manufacturing, and control strategy can be found in [11].

\section{B. Leg Design and Manufacturing}

The legs of SMoLBot-C are half circles with a diameter of $16 \mathrm{~mm}$ and 3D printed camshafts that form the connection between the DC motors and the C-legs (Fig. 2). The maximum possible $\mathrm{C}-\mathrm{leg}$ dimensions are calculated considering the primary goal of preventing any collision between two legs in two consecutive modules, especially while the robot is operating at a trot gait where two adjacent rear-front legs are $180^{\circ}$ apart in-phase and face a minimum gap.

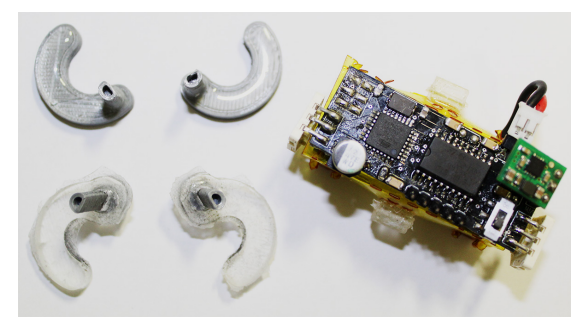

Fig. 2. Soft (PDMS) and 3D-printed (PLA coated with PDMS) C-legs with camshafts, and SMoLBot-C's single module.

This study investigated two types of legs: soft legs molded from PDMS and rigid legs 3D-printed from PLA. Soft legs are made with a mixture ratio of 1:7 and are cured for 2 hours at $65^{\circ} \mathrm{C}$ temperature [16]. Each soft leg weighs 1.5 grams. The camshafts are placed inside the leg molds before the curing process in order to increase the structural integrity of the connection between the PDMS C-leg and the 3D-printed camshaft. Rigid legs have the same design as the soft legs but manufactured from PLA using a 3D printer, and each weighs 0.80 grams. After the printing process, the 3D-printed rigid legs are coated with a thin layer of PDMS to match the soft legs' friction coefficients and the rigid legs. Therefore, the changes observed in the robot's locomotion between the soft and rigid legs would mainly be caused by the differences in the rigidity of the legs rather than the friction coefficients.

\section{FABRICATION AND ASSEMBLY}

The fabrication of the SMoLBot-C starts with laser cutting an acetate sheet with a laser engraver (Universal Laser systems, VLS 3.60) and then folding and embedding DC motors, LiPo battery, and PDMS locks into the modules. After finalizing the PCB and soldering the electrical components, the PCB is placed on top of each module and is fixed in place with holders. Figure 3 shows the assembled version of two modules, soft backbones and PDMS legs.

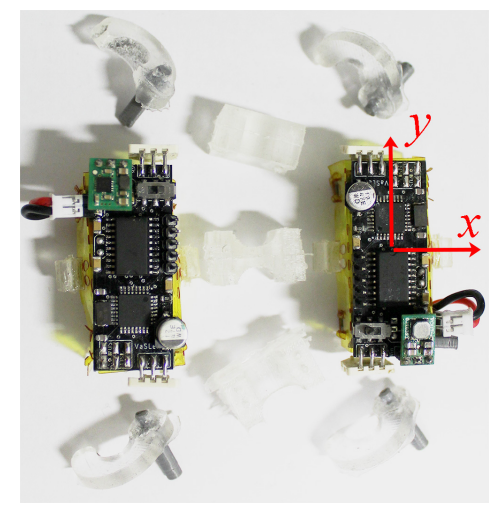

Fig. 3. Assembled modules, PDMS C-legs, and compliant backbones.

\section{RESUlts AND Discussion}

In order to investigate the effect of the legs' and backbones' compliances on the robot's locomotion, four different locomotion studies are conducted. First, the velocities of SMoLBot-Cs with rigid and soft legs and backbones are compared. Second, the obstacle climbing capability of SMoLBot-Cs with different backbones and leg types operating at a trot and pronk gait on rough terrain with obstacles is studied. Next, the mobility of the C-legged robots on an inclined surface is investigated. Last but not least, the effect of a passive tail on the miniature robot's locomotion while operating on smooth, rough, and inclined terrain is investigated. The last analysis demonstrates the advantageous and the detrimental effects of a compliant tail on the miniature robot's locomotion.

\section{A. Flat Ground}

In this study, the translational velocities of the robots are measured using the OptiTrack Motion Capture system, where the system captures all the dynamic parameters of the robot, such as the position of the center of gravity of the robot (COG), and the 3-D orientation of the robot. SMoLBot-C is run with $1,2,3$, and $4 \mathrm{~Hz}$ stepping frequency, and the robot's translational velocity is measured. At each stepping frequency, the error bar indicates the minimum and the maximum experimental velocity results between 5 trials. Fig. 4 illustrates the two-legged one-module SMoLBot-C's translational velocities with rigid and soft legs. It is important to note that the two-legged SMoLBot-C is not a bipedal robot, and a third point (rear part of the module) always touches the ground that makes the robot more similar to a 
differential drive robot. Translational velocity results shown in this manuscript are determined both in (BodyLength/s) and $(\mathrm{mm} / \mathrm{s})$, making it feasible to compare the multilegged robots' velocity with different numbers of modules. SMoLBot-C's DC motors can run up to $4 \mathrm{~Hz}$, which results in a maximum linear velocity of $160 \mathrm{~mm} / \mathrm{s}$ for the two-legged SMoLBot-C with soft legs operating at a trot gait. The linear velocity of $160 \mathrm{~mm} / \mathrm{s}$ is almost 8 body-length/s (Fig. 4), and it is the maximum velocity in bodylength/s units, compared to the other SMoLBot-Cs with more number of legs.

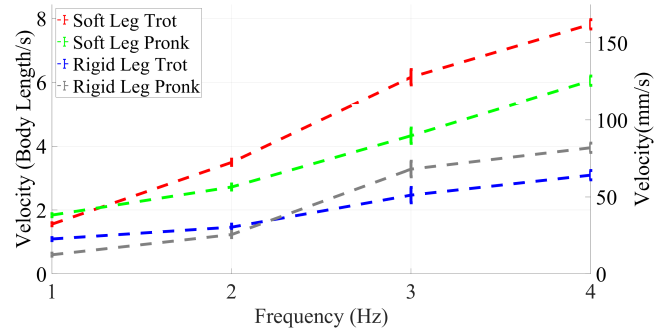

Fig. 4. Two-legged one-module SMoLBot-C with rigid and soft legs operating at trot and pronk gaits.

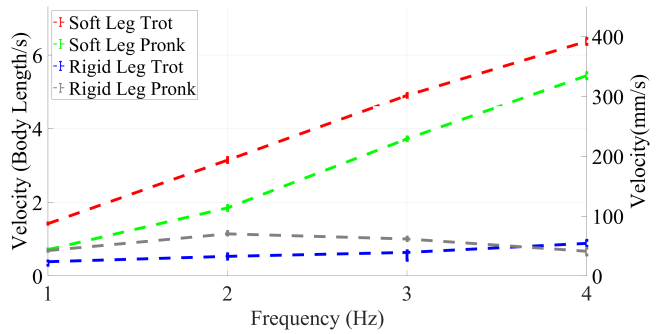

(a)

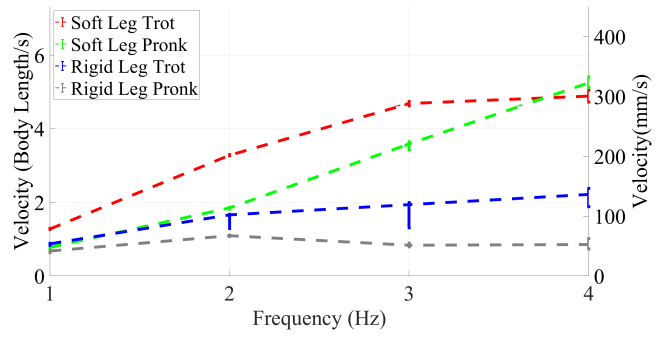

(b)

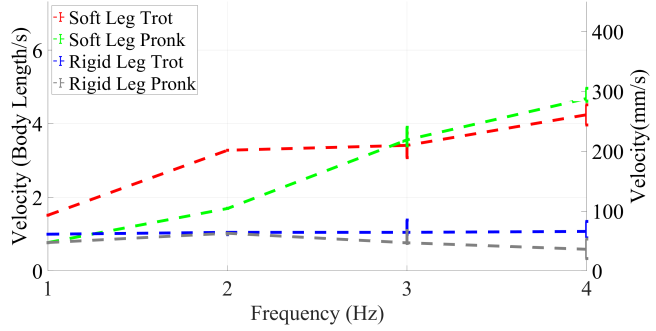

(c)

Fig. 5. Translational velocities of the four-legged two-module SMoLBot-C operating at trot and pronk gaits with (a) compliant-I, (b) with compliant-T, (c) and rigid backbones.

Figure 5 shows the translational velocities of the fourlegged SMoLBot-C with compliant-I, compliant-T, and rigid backbones. Velocity results indicate that the robot's velocity depends on the backbone stiffnesses, the number of modules, and type of the leg (soft or rigid). In every experiment, disregarding the module number and the backbone type, the robot with soft $\mathrm{C}$-legs possesses a velocity higher than the robot with rigid legs. Soft legs result in faster locomotion due to the soft material's elastic nature helping the leg conform to the surface better, compared to the similar-shaped rigid legs. Similarly, the compliant-I backbones introduce a higher degree of flexibility and conformation to the surface, which increases the robot's velocity. However, increasing the stepping frequency results in a slipping motion for the robots with rigid and compliant-T backbones, preventing further increase in the velocity.

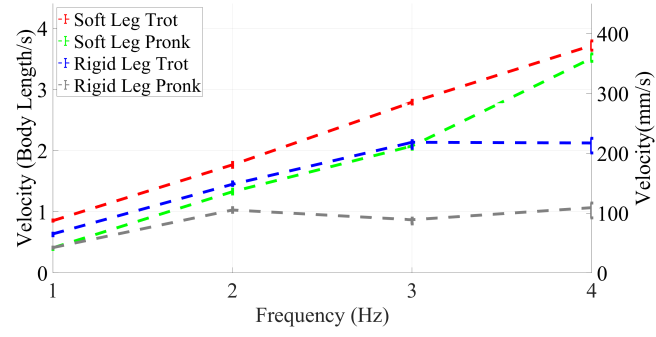

(a)

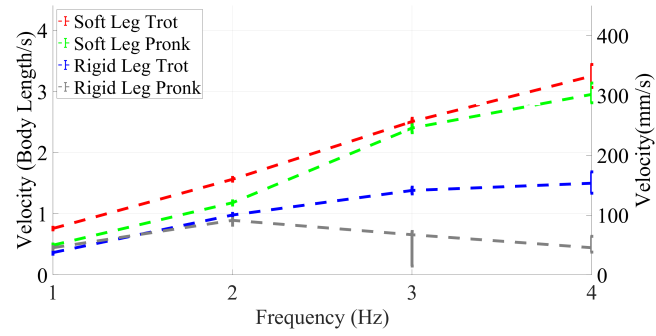

(b)

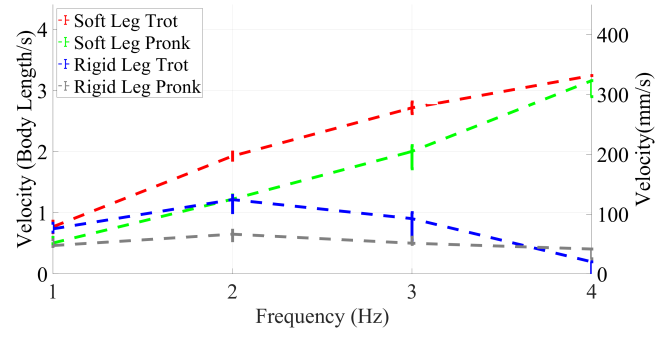

(c)

Fig. 6. Translational velocities of the six-legged three-module SMoLBot-C operating at trot and pronk gaits with (a) compliant-I, (b) with compliant-T, (c) and rigid backbones.

Figure 6 shows the velocity results for the six-legged threemodule SMoLBot-Cs. More modules added to the robot result in a statically-stable motion, despite the four-legged robots, where the robot falls on a third leg while operating at a trot gait. Statically-stable motion decreases the leg slip and increases the velocity of the robots with rigid legs. However, robots with compliant backbones and soft legs slip relatively less than the rigid cases; as a consequence, adding more legs to the robot with compliant backbones and soft legs do not change the velocity notably. Although, the velocities in 
body-length/s units significantly decrease for all the robots, due to the increase in the body length.

\section{B. Obstacle Traversal}

The next locomotion study investigates the mobility of the $\mathrm{C}$-legged SMoLBot on rough terrain with obstacles. Obstacle climbing experiments of SMoLBot-C are done at $2 \mathrm{~Hz}$ stepping frequency. These experiments are done with an experimental setup where the rigid obstacle's height is gradually increased until the robot fails to climb, and then the maximum height is measured. Tables I and II demonstrate the obstacle climbing experimental results of the SMoLBot$\mathrm{C}$, where the results indicate the maximum heights that the multi-legged robot can climb while operating with a trot or pronk gait. We observe that the robot with soft legs climbs up a larger obstacle due to the soft nature of the $\mathrm{C}$-legs that conform to the surface more and better support the robot during climbing. Similarly, another factor helping the robot to climb to a larger obstacle is body stiffness. Results show that SMoLBot-C with compliant-I backbones and soft legs can climb up a $24 \mathrm{~mm}$-high obstacle, which is almost 1.6 times the module's height. The robot is operating at a pronk gait, where all legs possess an equal phase and touch the obstacle simultaneously. These multiple instant contacts help the robot to form better support to initiate climbing, compared to a robot operating at a trot gait, where the two side-by-side legs are $180^{\circ}$ apart in phase, and the right and the left legs of the modules touch the obstacle once at a time. This periodical motion of the two side-by-side legs with $180^{\circ}$ phase difference limits the duration and the number of contact points with the obstacle, which decreases the maximum scalable obstacle height while operating with the trot gait.

TABLE I

Obstacle Climbing Experimental Results Of The Four-Legged Two-Module SMoLBot-C

\begin{tabular}{lccc}
\hline Gait/Leg & Compliant-I $(\mathrm{mm})$ & Compliant-T $(\mathrm{mm})$ & Rigid $(\mathrm{mm})$ \\
\hline Trot/Soft & 16.5 & 20.0 & 11.0 \\
Pronk/Soft & 24.0 & 20.0 & 13.0 \\
Trot/Rigid & 11.5 & 8.5 & 10.0 \\
Pronk/Rigid & 17.5 & 11.5 & 13.0 \\
\hline
\end{tabular}

Table II shows the obstacle climbing results of the sixlegged three-module robots operating with trot and pronk gaits. Increasing the number of legs helps the robot to climb to a higher obstacle. This can be explained considering the robot's locomotion with more legs; the robot with six legs contacts the ground at more locations at the rear part of the robot while the leader module (first module) is lifted. These more number of simultaneous striding legs, in conjunction with a better conformation of the legs to the ground, are achievable with compliant-I backbones, helping the robot climb easier to a higher obstacle. SMoLBot-C with compliant-I backbones operating at pronk gait climbs up to a height 2 times higher than a single module's height equal to $30 \mathrm{~mm}$.
TABLE II

Obstacle Climbing EXPerimental Results Of The SiX-LegGed THREE-MOdule SMOLBOT-C

\begin{tabular}{lccc}
\hline Gait/Leg & Compliant-I (mm) & Compliant-T $(\mathrm{mm})$ & Rigid (mm) \\
\hline Trot/Soft & 28.0 & 23.0 & 20.0 \\
Pronk/Soft & 30.0 & 26.0 & 24.0 \\
Trot/Rigid & 13.5 & 17.0 & 10.5 \\
Pronk/Rigid & 17.0 & 18.0 & 12.5 \\
\hline
\end{tabular}

\section{Inclined Surface}

Inclined surface experiments are done with an anglevarying inclined platform as in Fig. 7, that shows the time-lapse of a two-legged one-module SMoLBot-C on an inclined platform at $12^{\circ}$ and $20^{\circ}$ degrees. Table III shows the experimental results of the four-legged twomodule SMoLBot-Cs walking on an inclined surface at $2.5 \mathrm{~Hz}$ stepping frequency. Similar to the obstacle climbing experiments, the soft legs help the robot to conform to the surface better, providing the robot higher friction and better traction which indeed results in better locomotion on an inclined surface. Additionally, results indicate that decreasing the stiffnesses of the module connections increases the motions along $y$ direction of the individual module. This lateral motion increases the slip and decreases the mobility in an inclined surface, which is the case for the SMoLBot-C with compliant-I backbones; i.e. modules of the SMoLBot$\mathrm{C}$ with compliant-I backbones move along $y$ direction and the leader module (first module) slips along the $y$ direction preventing the robot to climb up (the axes are show in Fig. 3).

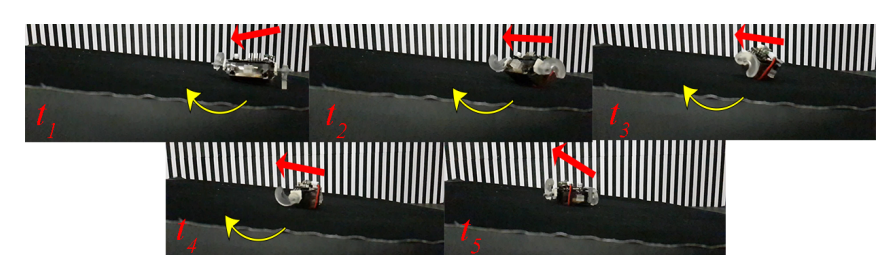

(a)

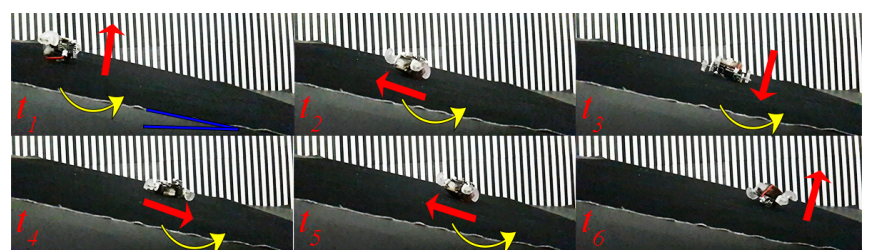

(b)

Fig. 7. (a) Two-legged one-module SMoLBot-C with soft legs climbing at trot gait and an inclination angle $12^{\circ}$. (b) Failed locomotion of the two-legged one-module SMoLBot-C with soft legs on a surface with an inclination angle of $20^{\circ}$. Red arrows show the direction of the head/front of the module, and yellow arcs show the COG's movement direction.

Table IV shows the maximum inclination angles of the six-legged three-module SMoLBot-Cs with compliant-I, compliant-T, and rigid backbones that the robot can walk up. Increasing the number of legs increases the number of contact points and helps the robot climb better. Six-legged three- 
module SMoLBot-C with compliant-T backbones operating at a trot gait can operate at a surface with an inclination angle of $38^{\circ}$ degrees. The higher walkable inclination angle of SMoLBot-C with compliant-T backbones can be explained considering single torsional DOF of compliant-T backbones providing movement in only $x$ direction without lateral $(y)$ movement; The legs conform to the surface better as the module rolls, helping the robot climb an inclined surface without slipping along $y$ direction.

TABLE III

INCLINED SURFACE EXPERIMENTAL RESULTS OF THE FouR-LEGged Two-Module SMOLBoT-C

\begin{tabular}{lccc}
\hline Gait/Leg & Compliant-I (deg) & Compliant-T (deg) & Rigid (deg) \\
\hline Trot/Soft & $25^{\circ}$ & $25^{\circ}$ & $23^{\circ}$ \\
Pronk/Soft & $28^{\circ}$ & $32^{\circ}$ & $22^{\circ}$ \\
Trot/Rigid & $9^{\circ}$ & $7^{\circ}$ & $8^{\circ}$ \\
Pronk/Rigid & $11^{\circ}$ & $12^{\circ}$ & $10^{\circ}$ \\
\hline
\end{tabular}

TABLE IV

INCLINED SURFACE EXPERIMENTAL RESULTS OF THE SIX-LEGgED Three-Module SMoLBot-C

\begin{tabular}{lccc}
\hline Gait/Leg & Compliant-I (deg) & Compliant-T (deg) & Rigid (deg) \\
\hline Trot/Soft & $32^{\circ}$ & $38^{\circ}$ & $33^{\circ}$ \\
Pronk/Soft & $28^{\circ}$ & $33^{\circ}$ & $23^{\circ}$ \\
Trot/Rigid & $15^{\circ}$ & $16^{\circ}$ & $13^{\circ}$ \\
Pronk/Rigid & $18^{\circ}$ & $19^{\circ}$ & $13^{\circ}$ \\
\hline
\end{tabular}

\section{Tail-Augmented Robot}

By means of improving the climbing capabilities, a tail is added to the robot, similar to the robot's biological inspirations where the tail plays a vital role by generating an additional contact (and a moment reaction) during climbing. Our results also show that adding a tail results in improved mobility on an inclined surface for $\mathrm{C}$-robots. To investigate the effect of a tail on the robot's locomotion, a passive tail, as shown in Fig. 8 is designed and assembled to the rear module of SMoLBot-C. A $50 \mathrm{~mm}$ long compliant tail made out of Thermo Plastic Elastomer (TPE) is attached to the PDMS lock at the last module's rear patch using metal pins. The locomotion study results of the two-legged one-module SMoLBot-C with a compliant tail and without a tail are shown in Table V.

The scalable obstacle height and inclination angle for a two-legged one-module robot without a tail are $3 \mathrm{~mm}$ and $12^{\circ}$, while these parameters for the robot with a compliant tail are $20 \mathrm{~mm}$ and $23^{\circ}$, respectively. The compliant tail forms additional support at the rear of the robot, while climbing an inclined surface or a rough terrain, helping the robot to climb higher; however, since the tail always is being dragged on the ground, it produces additional friction force, decreasing the velocity (Table V). This decreasing velocity behavior is observed in all multi-legged robots disregarding module number and backbone type while a passive tail is added to the robot (Tables V, VI, and VII velocity results).

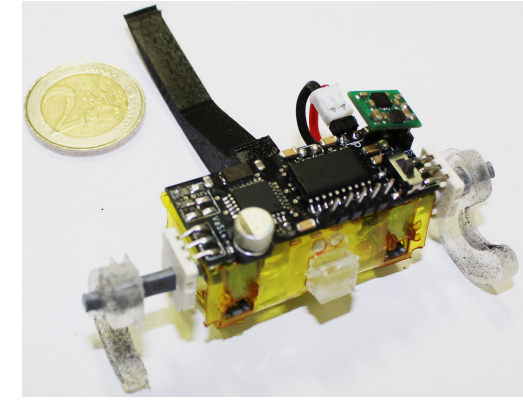

Fig. 8. Two-legged one-module SMoLBot-C with a 3D-printed compliant tail manufactured using a 3D printer from TPE.

TABLE V

Results Of The Two-Legged One-Module SMoLBot-C

\begin{tabular}{lcc}
\hline & With Tail & Without Tail \\
\hline Velocity at $2 \mathrm{~Hz}$ & $63(\mathrm{~mm} / \mathrm{s})$ & $72(\mathrm{~mm} / \mathrm{s})$ \\
Inclination angle & $23^{\circ}$ & $12^{\circ}$ \\
Scalable obstacle height & $20(\mathrm{~mm})$ & $3(\mathrm{~mm})$ \\
\hline
\end{tabular}

Tables VI and VII show the results of the four-legged and six-legged SMoLBot-Cs with and without tail, on smooth and rough terrains. Results show improvements in the robot's performance and robustness while a tail is assembled on the $\mathrm{C}$-legged robot on an inclined surface. Adding a tail to the multi-legged robot with rigid backbones improves the robot's mobility on rough terrain with obstacles, where the compliant tail is capable of deforming and forming a support for the rigid robot to climb over the obstacle. For instance, the ascent height of the four-legged and the six-legged robots with rigid backbones are increased from $13 \mathrm{~mm}$ to $23 \mathrm{~mm}$ and $24 \mathrm{~mm}$ to $27 \mathrm{~mm}$, respectively, while the tail is added to the rear modules. Conversely, adding a tail to the $\mathrm{C}$-legged robot with compliant-I backbones decreases the maximum ascent height. The tail added to the robot with compliant-I backbones lifts the last rear module while the leader (first) module is climbing the obstacle, and this lifting motion eliminates the feet-ground contacts and minimizes the stride force. Decreasing the legs' driving/striding forces explains the decreasing ascent height of the robot with compliant-I backbones.

TABLE VI

Locomotion Analysis Of The Four-Legged Two-Module SMoLBot-C With AND Without a TAIL

\begin{tabular}{lccc}
\hline Backbone & $\begin{array}{c}\text { Velocity } \\
(\mathrm{mm} / \mathrm{s})\end{array}$ & $\begin{array}{c}\text { Inclination angle } \\
(\mathrm{deg})\end{array}$ & $\begin{array}{c}\text { Obstacle height } \\
(\mathrm{mm})\end{array}$ \\
\hline Compliant-I: & & $34^{\circ}$ & 18 \\
With tail & 124 & $28^{\circ}$ & 24 \\
Without tail & 193 & & \\
\hline Compliant-T: & & $35^{\circ}$ & 22 \\
With tail & 172 & $32^{\circ}$ & 20 \\
Without tail & 200 & & \\
\hline Rigid: & & $25^{\circ}$ & 13 \\
With tail & 161 & $23^{\circ}$ & 23 \\
Without tail & 200 &
\end{tabular}


TABLE VII

Locomotion Analysis Of The SiX-Legged ThreE-Module SMoLBot-C With AND Without A TAIL

\begin{tabular}{lccc}
\hline Backbone & $\begin{array}{c}\text { Velocity } \\
(\mathrm{mm} / \mathrm{s})\end{array}$ & $\begin{array}{c}\text { Inclination angle } \\
(\mathrm{deg})\end{array}$ & $\begin{array}{c}\text { Obstacle height } \\
(\mathrm{mm})\end{array}$ \\
\hline Compliant-I: & 163 & $31^{\circ}$ & 24 \\
With tail & 180 & $32^{\circ}$ & 30 \\
Without tail & & & \\
\hline Compliant-T: & 165 & $40^{\circ}$ & 27 \\
With tail & 174 & $38^{\circ}$ & 25 \\
Without tail & & & 27 \\
\hline Rigid: & 156 & $37^{\circ}$ & 24 \\
With tail & 196 & $33^{\circ}$ & \\
Without tail & & & \\
\hline
\end{tabular}

\section{E. Discussion}

Comparing the robots' velocities on a flat ground shows that the two-legged one-module robot design possesses the highest velocity in the body-length/s unit, and the robot runs at 8 body-length/s velocity. In terms of linear $\mathrm{mm} / \mathrm{s}$ velocity, on a flat ground, the best result is obtained for a four-legged/soft-legs/trot gait/no-tail/compliant-I design configuration, that runs at $390 \mathrm{~mm} / \mathrm{s}$. Comparing the experiments' results on an inclined surface and a rough terrain shows that soft legs and backbones provide more support and a better conformation that helps the robot climb an obstacle and walk an inclined surface. For obstacle traversal experiments, the robot with six-legs/soft-legs/pronk gait/notail/compliant-I design parameters possesses the maximum climbable obstacle height, which is almost 2 times a module's height. For the inclined surface experiments, the robot design with six-legs/soft-legs/trot gait/with-tail/compliant-T possesses the highest inclination angle that SMoLBot-C can climb up. The robot with such design parameters can climb on an inclined surface with an inclination angle of $40^{\circ}$.

\section{CONCLUSION AND FUture WORK}

This paper investigates the locomotion behavior of a Clegged modular miniature robot (SMoLBot-C) with soft or rigid backbones on various terrain types. $\mathrm{C}-$ legged robots show robust and improved mobility on rough and inclined terrains compared to the pointy legged SMoLBot. This is due to a better surface conformation and continuous ground-feet contact. Results indicate that the $\mathrm{C}$-legged robot with soft legs and compliant-I backbones (lowest stiffnesses) possesses the maximum translational velocity than the other robots. Similarly, the PDMS legs' soft nature helps the robot climb over larger obstacles by forming better supports at the rear modules. Comparison of the SMoLBot-C results with four and six-legs indicates that the higher inclination angles and scalable obstacle heights can be achieved by increasing leg numbers; however, the robot's translational velocities are reduced in terms of BodyLength/s while more number of legs are added to the robot.

Studies on the robot maneuverability, gait analysis of Clegged robots, and finding efficient turning gaits are considered future work. Geometry optimization will be done to obtain a leg geometry that provides better performance in maximizing scalable obstacle height, the inclination angle, or the translational velocity.

\section{ACKNOWLEDGMENTS}

The authors would like to thank members of Bilkent Miniature Robotics Laboratory for their invaluable assistance throughout this project. This work is funded by the Scientific and Technological Research Council of Turkey (TUBITAK), Grant number: $116 \mathrm{E} 177$.

\section{REFERENCES}

[1] O. Ozcan, A. T. Baisch, D. Ithier, and R. J. Wood, "Powertrain selection for a biologically-inspired miniature quadruped robot," in 2014 IEEE International Conference on Robotics and Automation (ICRA). IEEE, 2014, pp. 2398-2405.

[2] G. Bai and B. Thomaszewski, "A c-legged monopedal robot and its transition from multiple locomotion modes," Journal of Mechanisms and Robotics, vol. 12, no. 4, 2020.

[3] A. F. Güç, M. A. İ. Kalin, C. Karakadioğlu, and O. Özcan, "C-quad: A miniature, foldable quadruped with c-shaped compliant legs," in 2017 IEEE International Conference on Robotics and Biomimetics (ROBIO). IEEE, 2017, pp. 26-31.

[4] S. Kawamura, D. Tanaka, T. Tanaka, D. Noguchi, Y. Hayakawa, M. Kaneko, K. Saito, and F. Uchikoba, "Neural networks ic controlled multi-legged walking mems robot with independent leg mechanism," Artificial Life and Robotics, vol. 23, pp. 380-386, 2018.

[5] D. Rus and M. T. Tolley, "Design, fabrication and control of origami robots," Nature Reviews Materials, vol. 3, no. 6, p. 101, 2018.

[6] P. Birkmeyer, K. Peterson, and R. Fearing, "Dash: A dynamic $16 \mathrm{~g}$ hexapedal robot," in 2009 IEEE/RSJ International Conference on Intelligent Robots and Systems, 2009, pp. 2683-2689.

[7] R. J. Wood, S. Avadhanula, R. Sahai, E. Steltz, and R. S. Fearing, "Microrobot design using fiber reinforced composites," Journal of Mechanical Design, vol. 130, no. 5, p. 052304, 2008.

[8] A. M. Hoover, E. Steltz, and R. S. Fearing, "Roach: An autonomous $2.4 \mathrm{~g}$ crawling hexapod robot," in 2008 IEEE/RSJ International Conference on Intelligent Robots and Systems. IEEE, 2008, pp. 2633.

[9] S. A. Rios, A. Fleming, and Y. K. Yong, "Miniature resonant ambulatory robot," IEEE Robotics and Automation Letters, vol. 2, no. 1, pp. 337-343, 2017.

[10] A. DeMario and J. Zhao, "A Miniature, 3D-Printed, Walking Robot With Soft Joints," ser. International Design Engineering Technical Conferences and Computers and Information in Engineering Conference, vol. Volume 5B: 41st Mechanisms and Robotics Conference, 2017. [Online]. Available: https://doi.org/10.1115/DETC2017-68182

[11] N. Mahkam, A. Bakir, and O. Özcan, "Miniature modular legged robot with compliant backbones," IEEE Robotics and Automation Letters, vol. 5, no. 3, pp. 3923-3930, 2020.

[12] E. V. Hoff, D. Jeong, and K. Lee, "Origamibot-i: A thread-actuated origami robot for manipulation and locomotion," in 2014 IEEE/RSJ International Conference on Intelligent Robots and Systems, 2014, pp. $1421-1426$.

[13] M. Askari, C. Karakadiouglu, F. Ayhan, and O. Ozcan, "MinIAQ-II: A miniature foldable quadruped with an improved leg mechanism," in IEEE International Conference on Robotics and Biomimetics (ROBIO'17), Macau, China, 12 2017, pp. 19-25.

[14] U. Saranli, M. Buehler, and D. E. Koditschek, "Rhex: A simple and highly mobile hexapod robot," The International Journal of Robotics Research, vol. 20, no. 7, pp. 616-631, 2001.

[15] K. Tadakuma, R. Tadakuma, A. Maruyama, E. Rohmer, K. Nagatani, K. Yoshida, A. Ming, M. Shimojo, M. Higashimori, and M. Kaneko, "Mechanical design of the wheel-leg hybrid mobile robot to realize a large wheel diameter," in 2010 IEEE/RSJ International Conference on Intelligent Robots and Systems, 2010, pp. 3358-3365.

[16] Z. Wang, A. A. Volinsky, and N. D. Gallant, "Crosslinking effect on polydimethylsiloxane elastic modulus measured by custom-built compression instrument," Journal of Applied Polymer Science, vol. 131, no. 22, 2014. 